this study we assess the contribution of early adulthood trajectories through education, employment or inactivity (age 16$24 y$ ) to cardiovascular health in mid-adulthood (age 46y).

Methods Participants are from BCS70 $(n=7,061)$ with data on education participation and economic activity during early adulthood and cardiometabolic outcomes at age 46y. Longitudinal latent class analysis was used to identify groups following different socioeconomic trajectories across ages 16-24y, based on participation in education, employment within different occupational classes, unemployment or inactivity. Cardiometabolic outcomes (waist circumference, systolic blood pressure (SBP), log-HDL cholesterol, log-triglycerides, HbA1c) at age 46 were regressed on early adulthood socioeconomic trajectory class, with and without adjustment for adult socioeconomic position (SEP) (age 46). All models were adjusted for sex, childhood SEP, adolescent health and early adulthood partnership and parenthood.

Results Six classes of early adulthood socioeconomic trajectory were identified, and labelled according to the primary activity engaged in: (1) Continued education, (2) Managerial employment, (3) Skilled non-manual employment, (4) Skilled manual employment, (5) Partly skilled employment, (6) Inactive. There was an increasing trend in waist circumference and triglycerides across classes 1-6 at age 46. Compared to the 'Continued education' class, waist circumference showed an increase of $1.87 \mathrm{~cm}(95 \% \mathrm{CI} 0.84,2.91)$ in class 2 , to $3.94 \mathrm{~cm}(95 \% \mathrm{CI}$ $2.35,5.52)$ in class 6 , and triglycerides an increase of $9.69 \%$ $(95 \%$ CI $3.26,16.53)$ in class 2 to $14.27 \%$ (95\%CI 3.88,25.7) in class 6. Compared to the 'Continued education' class, SBP was higher in classes 2-5, e.g. an increase of $1.18 \mathrm{mmHg}$ $(95 \%$ CI $0.06,2.29)$ in class 2 , and HDL cholesterol lower in classes $2-6$, e.g. $-3.51 \%(95 \% \mathrm{CI}-5.71,-1.25)$ in class 2 . No difference was seen in HbA1c levels across classes. Adjustment for occupational social class at age 46 resulted in only a small attenuation of these coefficients.

Conclusion Identification of socioeconomic trajectories allows assessment of socioeconomic exposures across the transitional period of early adulthood. These findings support the hypothesis that these exposures of early adulthood may contribute to development of behaviours or psycho-social factors which persist through adult life; further research is needed to understand these pathways, and the extent to which early adulthood socioeconomic trajectories are mediated by SEP in later life.

\section{OP74 ASSOCIATIONS OF FREE-LIVING SITTING TIME AND PHYSICAL ACTIVITY WITH GRIP STRENGTH AND STANDING BALANCE PERFORMANCE IN THE 1970 BRITISH COHORT STUDY}

${ }^{1} \mathrm{R}$ Cooper*, ${ }^{2} \mathrm{E}$ Stamatakis, ${ }^{3} \mathrm{~A}$ Sullivan, ${ }^{4} \mathrm{M}$ Hamer. ${ }^{1}$ Musculoskeletal Science and Sports Medicine Research Centre, Manchester Metropolitan University, Manchester, UK; ${ }^{2}$ Charles Perkins Centre, School of Public Health, University of Sydney, Sydney, Australia; ${ }^{3}$ Centre for Longitudinal Studies, UCL Institute of Education, London, UK; ${ }^{4}$ Institute of Sport Exercise and Health, UCL, London, UK

\subsection{6/jech-2020-SSMabstracts.73}

Background Many physical activity (PA) guidelines now include recommendations on strength and balance training, reflecting growing recognition of the importance of maintaining physical capability for healthy ageing. However, these recommendations are not widely communicated and are often targeted at older adults where most research evidence has been generated. Less well understood is whether there are benefits of greater levels of participation in physical activity and of reducing sedentary time for strength and balance earlier in adulthood. We therefore aimed to examine the associations of sitting time and time spent physically active with grip strength and standing balance performance in middle-age.

Methods A total of 4,726 men and women from the 1970 British Cohort study, with data on free-living sitting and activity (ascertained over 7 days using a thigh-mounted accelerometer (activPAL3 micro)), grip strength and standing balance performance at age 46 years were included in analyses. Linear and multinomial logistic regression models were used to test associations of sitting time, time spent in moderate-vigorous physical activity (MVPA) and total PA time with maximum grip strength and standing balance performance, respectively. Models were adjusted for wear time, sex, body mass index, height, disability, self-rated health, malaise, smoking and education.

Results Greater time spent sitting was associated with weaker grip strength in both sexes and this was maintained after adjustment for potential confounders and MVPA time (fullyadjusted regression coefficient: $-0.51 \mathrm{~kg}$ (95\% CI: -0.63 , 0.39 ) per 1 hour of sitting per day). Positive associations between total PA time and grip strength were observed in confounder-adjusted models but were fully attenuated after adjustment for sitting time. There was only weak evidence of an association between sitting time and balance performance. However, there was evidence to suggest that participants who spent more time in MVPA and total PA had higher relative risks of successfully balancing for 30 seconds with their eyes closed (vs poor balance performance). However, these associations were not maintained after adjustment for potential confounders.

Conclusion In a national sample of middle-aged adults there was a robust association between greater time spent sitting, measured using a gold-standard assessment, and muscle weakness. Associations of PA time with grip strength and balance performance were attenuated in fully-adjusted models. This highlights the potential importance of promoting less sitting and activities that specifically benefit muscle strength and balance performance in midlife to ensure that people maintain all important aspects of their physical capability as they age.

\section{Friday 11 September}

\section{Non-Communicable Disease: Treatment}

\section{OP75 THE POTENTIAL IMPACT OF COGNITIVE REHABILITATION ON THE FUTURE BURDEN OF POST- STROKE COGNITIVE IMPAIRMENT IN IRELAND TO 2035: PRELIMINARY RESULTS USING A MODEL-BASED APPROACH}

${ }^{1}$ E Sexton* ${ }^{1}$ NA Merriman, ${ }^{2} \mathrm{NA}$ Donnelly, ${ }^{2} \mathrm{MA}$ Wren, ${ }^{3} \mathrm{P}$ Bandosz, ${ }^{3} \mathrm{M}$ Guzman-Castillo, ${ }^{3} \mathrm{M}$ O'Flaherty, ${ }^{1} \mathrm{~A}$ Hickey, ${ }^{4} \mathrm{~K}$ Bennett. ${ }^{1}$ Dept of Psychology, Royal College of Surgeons in Ireland, Dublin, Ireland; ' Division of Social Research, Economic and Social Research Institute, Dublin, Ireland; ${ }^{3}$ Dept of Public Health and Policy, University of Liverpool, Liverpool, UKi ${ }^{4}$ Division of Population Health, Royal College of Surgeons in Ireland, Dublin, Ireland

\subsection{6/jech-2020-SSMabstracts.74}

Background Post-stroke cognitive impairment (PSCI) is a frequent consequence of stroke, and reduces quality of life and 